\title{
Light-Activated Hydrogel Formation via the Triggered Folding and Self-Assembly of a Designed Peptide
}

\author{
Lisa A. Haines ${ }^{\dagger}$, Karthikan Rajagopal ${ }^{\dagger}$, Bulent Ozbas ${ }^{\ddagger}$, Daphne A. Salick ${ }^{\dagger}$, Darrin J. \\ Pochan ${ }^{*} \neq$, and Joel P. Schneider ${ }^{\star}, \dagger$ \\ $\dagger$ Department of Chemistry and Biochemistry, University of Delaware, Newark, Delaware 19716-2522 \\ $\$$ Materials Science and Engineering, Delaware Biotechnology Institute, University of Delaware, Newark, \\ Delaware 19716-2522
}

\section{Abstract}

Photopolymerization can be used to construct materials with precise temporal and spatial resolution. Applications such as tissue engineering, drug delivery, the fabrication of microfluidic devices and the preparation of high-density cell arrays employ hydrogel materials that are often prepared by this technique. Current photopolymerization strategies used to prepare hydrogels employ photoinitiators, many of which are cytotoxic and require large macromolecular precursors that need to be functionalized with moieties capable of undergoing radical cross-linking reactions. We have developed a simple light-activated hydrogelation system that employs a designed peptide whose ability to self-assemble into hydrogel material is dependent on its intramolecular folded conformational state. An iterative design strategy afforded MAX7CNB, a photocaged peptide that, when dissolved in aqueous medium, remains unfolded and unable to self-assemble; a $2 \mathrm{wt} \%$ solution of freely soluble unfolded peptide is stable to ambient light and has the viscosity of water. Irradiation of the solution $(260<\lambda<360 \mathrm{~nm})$ releases the photocage and triggers peptide folding to produce amphiphilic $\beta$-hairpins that self-assemble into viscoelastic hydrogel material. Circular dichroic (CD) spectroscopy supports this folding and self-assembly mechanism, and oscillatory rheology shows that the resulting hydrogel is mechanically rigid ( $G^{\prime}=1000 \mathrm{~Pa}$ ). Laser scanning confocal microscopy imaging of NIH 3T3 fibroblasts seeded onto the gel indicates that the gel surface is noncytotoxic, conducive to cell adhesion, and allows cell migration. Lastly, thymidine incorporation assays show that cells seeded onto decaged hydrogel proliferate at a rate equivalent to cells seeded onto a tissue culture-treated polystyrene control surface.

Photopolymerization is extensively used in the fabrication of a diverse array of materials that include industrial membranes and coatings, ${ }^{1}$ dental adhesives, ${ }^{2}$ and optical and electronic materials. ${ }^{1}$ The use of light to initiate polymerization is now finding use in the construction of hydrogel materials, dilute polymer networks capable of encapsulating a large volume of water. 3,4 Light-derived hydrogels are useful materials having broad biomedical applications that include drug delivery, ${ }^{5-8}$ wound healing 9,10 tissue engineering ${ }^{11-14}$ and construction of high-density cell arrays. ${ }^{15-17}$ In addition, hydrogels are extensively used in the fabrication of contact lenses ${ }^{18,19}$ and microfluidic devices serving as environmentally sensitive channel dams. ${ }^{20-22}$ Irrespective of the final application, photopolymerization allows hydrogel material to be formed with both temporal and spatial resolution, whether in a targeted body cavity or the strict confines of a microfluidic channel.

E-mail: schneijp@udel.edu.

Supporting Information Available: Experimentals, analytical HPLC and ESI and MALDI-TOF mass spectra of peptides. This material is available free of charge via the Internet at http://pubs.acs.org. 
Typically, photopolymerized hydrogels are prepared via radical chemistry in which a solution of macromolecular precursor, a preformed water-soluble polymer containing reactive groups that can be cross-linked, and a photoinitiator are exposed to light. Radical generation and subsequent polymer cross-linking produces hydrogel material. With respect to biomedical applications, using macromolecular precursors to form the gel is favored over the direct polymerization of small molecule monomers to circumvent toxicity issues inherent to many commercially available monomers. ${ }^{4}$ In microfluidic devices, macromolecular precursors are used to better control the mechanical properties of the final hydrogel since the extent and type of cross-linking can be programmed at the level of precursor before material formation is initiated. Precursors are normally prepared by derivatizing an existing polymer with functional groups capable of undergoing radical cross-linking reactions. For example, acrylated poly (ethylene glycol)s 16 and poly(vinyl alcohol)s ${ }^{23}$ as well as cinnamated hyaluronic acids ${ }^{24}$ have been used. Not only is the design of the precursor important for the success of a particular application but also the choice of photoinitiator can be crucial. In vivo applications demand that the initiator be cytocompatible; however, many common initiators are cytotoxic at low concentrations or display cytotoxic behavior that is highly dependent on concentration. 11,25 In addition, most initiators are only sparingly soluble in water and must be dissolved in an organic solvent prior to use. ${ }^{11}$ However, carefully assessing initiator cytoxicity as a function of concentration, chemical additives, and light intensity can lead to useful systems. For example, Anseth and co-workers ${ }^{11}$ have reported that, although sparingly soluble in water, 2 hydroxy-1-[4-(hydroxyethoxy)phenyl]-2-methyl-1-propanone and camphorquinone (UV and visible light initiators, respectively) show efficient reactivity yet are cytocompatible under appropriate environmental conditions. Although elegant work is ongoing, there exists a large opportunity for alternate light-activated strategies that produce cytocompatible hydrogels with controlled mechanical and morphological properties.

We sought to develop a simple light-activated hydrogel system that did not involve the use of macromolecular precursors or photoinitiators. This new system employs light to initiate the self-assembly of water-soluble peptides into hydrogel material. Specifically, light is used to trigger the folding of a small peptide into an amphiphilic $\beta$-hairpin conformation, a structure highly amenable to efficient self-assembly into mechanically rigid hydrogel (Figure 1). Central to this hydrogelation strategy is the design of MAX7CNB, a 20-residue photocaged peptide that remains unfolded in aqueous solution under ambient light. However, exposure to UV radiation results in an uncaged peptide that intramolecularly folds. Folded hairpins are designed to undergo both facial self-assembly, via the association of their hydrophobic valine-rich faces and lateral assembly, via the formation of intermolecular hydrogen bonds between neighboring hairpins (both intra- and intermolecular H-bonds are shown in black in Figure 1). Together, these self-assembly events lead to a hydrogel having a nanostructure composed of a network of bilayer hairpin fibrils where noncovalent physical interfibril cross-links are formed by the hydrophobic collapse of orthogonal hairpins (Figure 1a). Extensive transmission electron microscopy and small-angle neutron scattering studies of hydrogel networks formed from similar $\beta$-hairpins that fold and self-assemble into hydrogel when triggered by changes in $\mathrm{pH}$, 26 ionic strength, ${ }^{27}$ and temperature ${ }^{28}$ support this proposed model of nanostructure.

\section{Results and Discussion}

MAX7CNB was conceived through an iterative design process that began with an earlier design, MAX1. This 20 -residue peptide is composed of high $\beta$-sheet propensity valine and lysine residues ${ }^{29-31}$ flanking a central tetrapeptide (-VDPPT-) designed to adopt type II' turn structure (Figure 1b). ${ }^{32-34}$ The ability of MAX1 to assemble is dependent on its unimolecular folded state. Under acidic conditions, the lysine side chains are protonated, which prevents the peptide from folding and, thus, self-assembling. Unfolded MAX1 is highly soluble, and 1-2 wt $\%$ solutions display rheological properties similar to those of water. Intramolecular folding 
and consequent self-assembly can be triggered by either screening some of the lysine-based charge with the addition of salt at physiological $\mathrm{pH}^{27}$ or by neutralizing the charge by increasing the $\mathrm{pH}$ of low ionic strength solutions to 9.26

Folded MAX1 hairpins are amphiphilic, where one face of the hairpin is lined with hydrophobic valine residues and the other face is lined with hydrophilic lysine residues, similar to the folded hairpin shown in Figure 1a. In addition to electrostatic interactions, previous studies indicated that the formation of both intra- and intermolecular hydrophobic interactions between valine residues is a key factor in promoting folding and selfassembly, respectively. ${ }^{28,35} \mathrm{We}$ envisioned that incorporating an amino acid residue bearing a charged side chain on the hydrophobic face would disrupt these hydrophobic interactions, thereby inhibiting the folding and self-assembly of the peptide. If so, a triggering mechanism could then be devised where this unwanted charge could be removed by UV illumination, allowing the peptide to fold and assemble into a hydrogel on cue.

MAX6 was initially prepared to test whether a charged group incorporated at the hydrophobic face would prevent folding. MAX6 is identical in sequence to MAX1 except for the alteration of valine at position 16 to glutamic acid, a carboxylate-bearing residue (Figure 1b). In the folded state, the glutamic acid side chain would be displayed in the center of the hydrophobic face. Figure 2 shows the CD spectrum of MAX6 under solution conditions at which MAX1 readily folds and selfassembles into hydrogel $\left(\mathrm{pH} \mathrm{9,37}{ }^{\circ} \mathrm{C}\right) .{ }^{28}$ As evident by the random coil signature, MAX6 is unfolded, indicating that the inclusion of negative charge on the hydrophobic face successfully inhibits folding.

On the basis of this result, incorporation of a residue modified with a charged, photosensitive group on its side chain at position 16 should prohibit folding, self-assembly, and ultimate material formation. An additional design requirement is that, after decaging, the resultant side chain must allow folding and self-assembly to occur. Cysteine residues are attractive for modification since their thiol side chains are not only relatively hydrophobic but also sufficiently nucleophilic and therefore easily functionalized. Although the unperturbed $\mathrm{p} K_{\mathrm{a}}$ of cysteine is about 8.5 (resulting in a significant population of deprotonated side chains at $\mathrm{pH} 9$, which could actually hinder folding), we envisioned that this residue's $\mathrm{p} K_{\mathrm{a}}$ would be shifted to higher values since its side chain resides in an extremely hydrophobic environment in the folded and self-assembled state. In addition, intermolecular disulfide bond formation after decaging is also a possibility that could enhance self-assembly. MAX7 was prepared to determine whether including a cysteine at position 16 would still allow the peptide to fold and self-assemble. The CD spectrum of MAX7 (Figure 2), which is nearly identical to that of MAX1 (not shown for clarity), shows a clear minimum at $215 \mathrm{~nm}$, consistent with $\beta$-sheet structure, demonstrating that this peptide readily folds and self-assembles into $\beta$-sheet-rich structures under the solution conditions where MAX6 remains unfolded. At low peptide concentrations (micromolar) such as those used for the CD studies, there is not enough peptide to gel the water present in the aqueous buffer. However, peptide folding and self-assembly still occurs, affording aggregates that are not large enough to appreciably scatter light, making CD measurements possible. Importantly, these data demonstrates that cysteine's side chain is conducive to folding and self-assembly.

The final design element entails the choice of photocage. Although a plethora of potential photoactive groups exists, the $\alpha$-carboxy-2-nitrobenzyl cage (Figure 1b) was employed because it bears a negative charge at neutral to basic $\mathrm{pH}$, has a respectable quantum yield for decaging, 36,37 and forms a noncytotoxic byproduct upon photolysis. 38 The final peptide MAX7CNB was prepared by reacting MAX7 with 2-bromo-2-(2-nitrophenyl)acetic acid via a modified protocol originally reported by Bayley and co-workers. ${ }^{39}$ Figure 3 shows CD spectra of a $150 \mu \mathrm{M}$ solution of MAX7CNB under basic conditions after 1 and $13 \mathrm{~h}$. The 
random coil signature remains nearly invariant, demonstrating that the cage inhibits peptide folding even after extended periods of time at $37^{\circ} \mathrm{C}$. Finally, exposure of the same solution (contained within a 1 mmpath length quartz cell) to UV light $(\lambda>300 \mathrm{~nm}$ ) for $30 \mathrm{~min}$ from a hand-held lamp results in decaging and peptide folding/self-assembly as evident by the $\beta$-sheet signature of the CD spectrum (Figure 3).

Quantitative decaging was verified by reverse-phase (RP) HPLC and mass spectroscopic analysis of the CD sample (data not shown).

At higher peptide concentrations ( $2 \mathrm{wt} \%, \sim 6 \mathrm{mM}$ ), where MAX1 is known to form a rigid hydrogel, an aqueous solution of MAX7CNB is freely flowing, displaying the viscosity of water at basic $\mathrm{pH}$. However, exposure of this solution to UV for $1 \mathrm{~h}$ results in the formation of a rigid hydrogel. In our experiments, MAX7CNB solutions are prepared in simple glass vials, resulting in rather large solution column heights $(\sim 0.3 \mathrm{~cm})$; as a result, efficient decaging takes about an hour. Photoinduced hydrogelation within a microfluidic device should be much more expedient since column heights on the order of micrometers are normally encountered. ${ }^{21}$ Figure 4 compares frequency sweep oscillatory rheology data obtained from 2 wt $\%$ preparations of MAX7 (having never been caged) and MAX7CNB after decaging. This experiment measures the storage modulus $\left(G^{\prime}\right)$, a measure of a material's elastic response (rigidity) to varying frequencies of applied oscillatory stress. The insensitivity of $G^{\prime}$ to frequency for both MAX7 and decaged MAX7CNB demonstrates that these gels are heavily cross-linked. ${ }^{26}$ The cross-links are permanent in nature yet are formed noncovalently via hydrophobic association of $\beta$-hairpins in the self-assembled state. More importantly, the magnitude of the observed storage modulus for the decaged hydrogel is approximately 1000 $\mathrm{Pa}$, making it a significantly rigid hydrogel material. The storage modulus of decaged MAX7CNB is slightly lower than that observed for MAX7, probably due to an effect of the 2-nitrosoglyoxylic byproduct formed during the decaging event. The loss modulus $\left(G^{\prime \prime}\right)$, a measure of a material's viscous response, was also measured as a function of varying frequencies of applied oscillatory stress. $G^{\prime \prime}$ values were more than an order of magnitude lower than the corresponding $G^{\prime}$ values for each gel, again indicating that the gels were quite rigid. The inset in Figure 4 shows the self-supporting nature of 2 wt \% MAX7 and decaged MAX7CNB hydrogels. The yellow coloration in the decaged sample is due to the benign photocage byproduct. The combination of the $\mathrm{CD}$ results in Figure 3 and the rheology data in Figure 4 indicates that peptide folding and consequent self-assembly does not occur until UV radiation activates decaging.

The oxidation state of the cysteine residue of decaged MAX7CNB in the hydrogel was difficult to directly measure since the 2-nitrosoglyoxylic byproduct formed during the decaging event absorbs in the same region as many colorimetric probes used to measure free thiol content. However, a hydrogel formed from uncaged MAX7 that had been subjected to decaging conditions (e.g., the clear hydrogel pictured in the inset of Figure 4) was analyzed by a 2,2'dithiodipyridine assay. ${ }^{40}$ The extent of oxidation was determined independently for three samples, and in each case approximately $60 \%$ of the hairpins were oxidized with very little variation among the samples. An extensive polymeric network of disulfide bonds is not possible since the peptide contains only one cysteine. In fact, the largest possible disulfide-bonded aggregate is a dimer. Matrix-assisted laser desorption ionization time-of-flight (MALDI-TOF) mass spectrometry of MAX7 hydrogel shows exclusively monomer and disulfided dimer (see Supporting Information). It is currently unknown whether the oxidation state of MAX7 influences the rheological properties of the hydrogel since, in our hands, it is nearly impossible to keep the peptide reduced under the conditions of the experiment.

Finally, future applications such as tissue engineering or the fabrication of high-density cell arrays demand that the hydrogel surface be cytocompatible. The cytocompatibility of the 
decaged MAX7CNB hydrogel surface was assessed by employing a live/dead assay where NIH 3 T3 fibroblasts were seeded onto the gel after light activation and equilibration with Dulbecco's modified Eagle cell culture medium ( $\mathrm{pH}$ 7.4) containing serum. Cells were incubated on the surface for $24 \mathrm{~h}$, enough time to allow the cells to attach to the surface and adopt healthy spread-out morphologies via the formation of actin stress fibers. Figure 5a shows a laser scanning confocal microscopy (LSCM) image of cells maintained on $2 \mathrm{wt} \%$ decaged gel after fluorescent dyes have been added that specifically dye living cells (green) and dead cells (red). Also, shown for comparison are cells that have been seeded onto a sterile borosilicate control surface under identical conditions (Figure 5b). These reproducible images demonstrate that the gel surface is noncytotoxic. Figure $5 \mathrm{c}$ shows cells that have been stained with both a DNA-specific fluorescent dye (4',6-diamidino-2-phenylindole, blue) and a fluorescent dye specific for F-actin (Alexa fluor 488 phalloidin, green). Cells undergoing surface adhesion events form stress fibers, lamel-lipodia and filopodia, all of which contain Factin. These structures help define the cellular morphology and aid locomotion. ${ }^{41}$ The formation of lamellipodia (flattened projections from cells) and filopodia (thin extensions from cells) are clearly seen in Figure 5c; the inset shows a magnified fibroblast where thin filopodia are more clearly observed. Actin stress fibers (not labeled for clarity) can be seen crossing over the nuclei of the cells. Also evident in the image are cells that contain ruffled edges; fibroblasts undergoing migration ruffle their membranes as they pull themselves across a given substratum. ${ }^{41}$ Together, the microscopic data demonstrate that the gel surface is noncytotoxic and is conducive to cell adhesion and allows migration. Last, cell proliferation was monitored over $72 \mathrm{~h}$ by measuring $\left[{ }^{3} \mathrm{H}\right]$ thymidine uptake as a function of time (Figure $5 \mathrm{~d}$ ). In this experiment, fibroblasts (20 000 cells/well) were seeded onto 2 wt \% MAX7CNB hydrogels (after exposure to light and equilibration with cell culture medium) and onto control tissue culture-treated polystyrene surfaces. The data show that cells proliferate on the hydrogel surface at nearly the same rate as the control surface and that the photocage byproduct, 2nitrosoglyoxylic acid, minimally influences proliferation.

For the cell-based experiments described here, hydrogelation was initiated at $\mathrm{pH} 9$ and cell culture medium was added to the resulting gels, effectively adjusting the $\mathrm{pH}$ to 7.4. Although this methodology is conducive to the fabrication of high-density cell arrays, many tissue engineering applications necessitate that cells be incorporated within the three-dimensional network of the hydrogel with serum components present. For these applications, a caged peptide capable of undergoing light-induced folding and self-assembly at pH 7.4 is needed; we are currently developing such a peptide.

\section{Conclusions}

Our approach to hydrogel formation entails the triggered folding and consequent self-assembly of $\beta$-hairpin peptides. We had earlier demonstrated that peptides can be designed to undergo triggered hydrogelation in response to changes in solution $\mathrm{pH},{ }^{26}$ ionic strength, ${ }^{27}$ or temperature. ${ }^{28}$ Herein, we show that light can be used as a trigger to initiate peptide folding and consequent self-assembly. We achieve this by covalently incorporating a cytocompatible photocleavable moiety that electrostatically inhibits peptide folding and, therefore, the intermolecular self-assembly process that leads to hydrogel formation. However, when irradiated, the photocage is released to initiate folding, self-assembly, and material formation to produce a mechanically rigid, noncytotoxic hydrogel. The utility of this novel photoinitiated hydrogelation mechanism is currently being investigated in our lab for use in applications that demand spatially and temporally resolved material formation. 


\section{References}

1. Hughes, LJ.; Hughes, LA. Handbook of polymer coatings for electronics. Vol. 2nd ed.. Park Ridge, NJ: Noyes Publications; 1990. p. 178-192.

2. Moszner N, Salz U. Prog. Polym. Sci 2001;26:535-576.

3. Fisher JP, Dean D, Engel PS, Mikos AG. Annu. Rev Mater. Res 2001;31:171-181.

4. Nguyen KT, West JL. Biomaterials 2002;23:4307-4314. [PubMed: 12219820]

5. Hill-West JL, Dunn RC, Hubbell JA. J. Surl. Res 1995;59:759-763.

6. An YJ, Hubbell JA. J. Controlled Release 2000;64:205-215.

7. Elisseeff J, Anseth K, Sims D, McIntosh W, Randolph M, Langer R. Proc. Natl. Acad. Sci. U.S.A 1999;96:3104-3107. [PubMed: 10077644]

8. Burkoth AK, Burdick J, Anseth KS. J. Biomed. Mater. Res 2000;51:352-359. [PubMed: 10880076]

9. West JL, Hubbell JA. Proc. Natl. Acad. Sci. U.S.A 1996;93:13188-13193. [PubMed: 8917566]

10. Hill-West JL, Chowdhury SM, Sawhney AS, Pathak CP, Dunn RC, Hubbell JA. Obstet. Gynecol. (N.Y.) 1994;83:59-64.

11. Bryant SJ, Nuttelman CR, Anseth KS. J. Biomater. Sci._Polym. Ed 2000;11:439-457. [PubMed: 10896041]

12. Elisseeff J, McIntosh W, Anseth K, Riley S, Ragan P, Langer R. J. Biomed. Mater. Res 2000;51:164171. [PubMed: 10825215]

13. Burdick JA, Chung C, Jia XQ, Randolph MA, Langer R. Biomacromolecules 2005;6:386-391. [PubMed: 15638543]

14. Zhang ZY, Shum P, Yates M, Messersmith PB, Thompson DH. Bioconjugate Chem 2002;13:640646.

15. Revzin A, Sekine K, Sin A, Tompkins RG, Toner M. Lab-on-a-Chip 2005;5:30-37. [PubMed: 15616737]

16. Revzin A, Tompkins RG, Toner M. Langmuir 2003;19:9855-9862.

17. Suh KY, Seong J, Khademhosseini A, Laibinis PE, Langer R. Biomaterials 2004;25:557-563. [PubMed: 14585705]

18. Wichterle O, Lim D. Nature 1960;185:117-118.

19. McMahon TT, Zadnik K. Cornea 2000;19:730-740. [PubMed: 11009325]

20. Eddington DT, Beebe DJ. Adv. Drug Delivery Rev 2004;56:199-210.

21. Beebe DJ, Moore JS, Bauer JM, Yu Q, Liu RH, Devadoss C, Jo BH. Nature 2000;404:588-590. [PubMed: 10766238]

22. Burdick JA, Khademhosseini A, Langer R. Langmuir 2004;20:5153-5156. [PubMed: 15986641]

23. Martens P, Anseth KS. Polymer 2000;41:7715-7722.

24. Miyamoto K, Sasaki M, Minamisawa Y, Kurahashi Y, Kano H, Ishikawa S. J. Biomed. Mater. Res 2004;70A:550-559.

25. Hanks CT, Strawn SE, Wataha JC, Craig RG. J. Dent. Res 1991;70:1450-1455. [PubMed: 1835727]

26. Schneider JP, Pochan DJ, Ozbas B, Rajagopal K, Pakstis L, Kretsinger J. J. Am. Chem. Soc 2002;124:15030-15037. [PubMed: 12475347]

27. Ozbas B, Kretsinger J, Rajagopal K, Schneider JP, Pochan DJ. Macromolecules 2004;37:7331-7337.

28. Pochan DJ, Schneider JP, Kretsinger J, Ozbas B, Rajagopal K, Haines L. J. Am. Chem. Soc 2003;125:11802-11803. [PubMed: 14505386]

29. Brack A, Orgel LE. Nature 1975;256:383-387. [PubMed: 238134]

30. Kim CA, Berg JM. Nature 1993;362:267-270. [PubMed: 8459852]

31. Minor DL Jr, Kim PS. Nature 1994;367:660-663. [PubMed: 8107853]

32. Nair CM, Vijayan M, Venkatachalapathi YV, Balaram P. J. Chem. Soc., Chem. Commun 1979:11831184.

33. Bean JW, Kopple KD, Peishoff CE. J. Am. Chem. Soc 1992;114:5328-5334.

34. Stanger HE, Gellman SH. J. Am. Chem. Soc 1998;120:4236-4237.

35. Rajagopal K, Ozbas B, Pochan DJ, Schneider JP. Eur. Biophys. J. in press 
36. Pan P, Bayley H. FEBS Lett 1997;405:81-85. [PubMed: 9094429]

37. McCray JA, Trentham DR. Annu. Rev. Biophys. Biophys. Chem 1989;18:239-270. [PubMed: 2660825]

38. Walker JW, Gilbert SH, Drummond RM, Yamada M, Sreekumar R, Carraway RE, Ikebe M, Fay FS. Proc. Natl. Acad. Sci. U.S.A 1998;95:1568-1573. [PubMed: 9465056]

39. Chang CY, Niblack B, Walker B, Bayley H. Chem. Biol 1995;2:391-400. [PubMed: 9383441]

40. Pedersen AO, Jacobsen J. Eur. J. Biochem 1980;106:291-295. [PubMed: 7341229]

41. Mitchison TJ, Cramer LP. Cell 1996;84:371-379. [PubMed: 8608590]

\section{Acknowledgment}

We thank the NIH (R01 DE016386-01) for support. We also thank Ann Snellinger for collecting mass spectral data and Professor John Koh for discussion concerning cell culturing. 


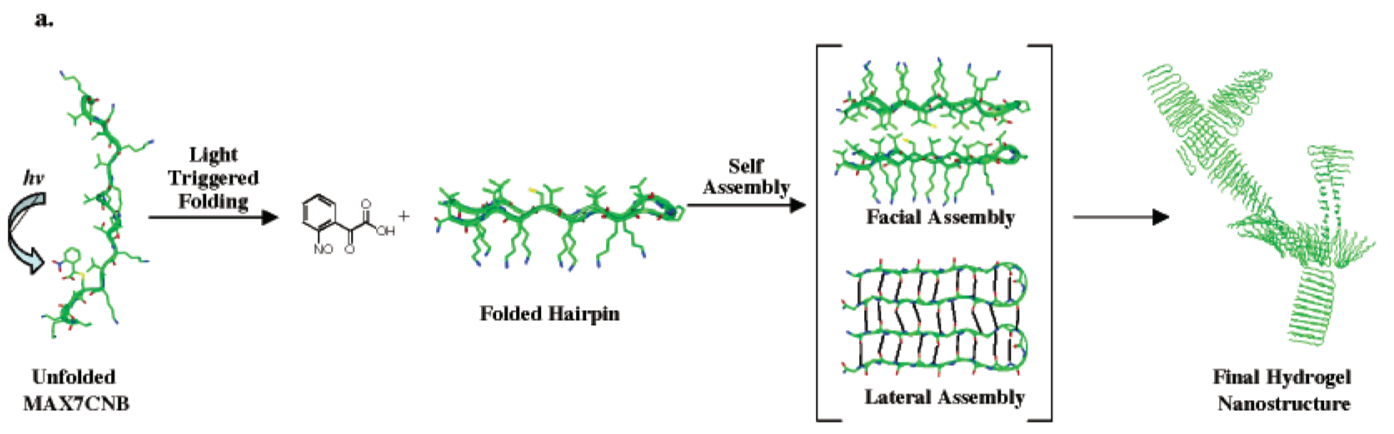

b.

Sequence

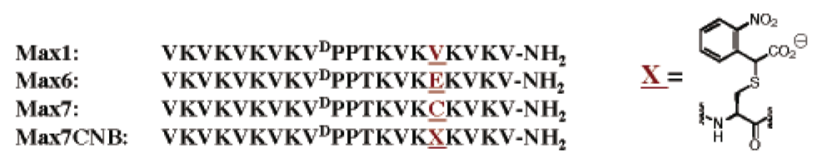

Figure 1.

(a) Light-induced material formation. UV illumination of MAX7CNB results in side-chain decaging and $\beta$-hairpin intramolecular folding. Subsequent facial and lateral self-assembly ultimately affords hydrogel material. (b) Sequences of carboxyamidated peptides. $\alpha$ Carboxy-2-nitrobenzyl protection of the cysteine in MAX7 affords MAX7CNB. 


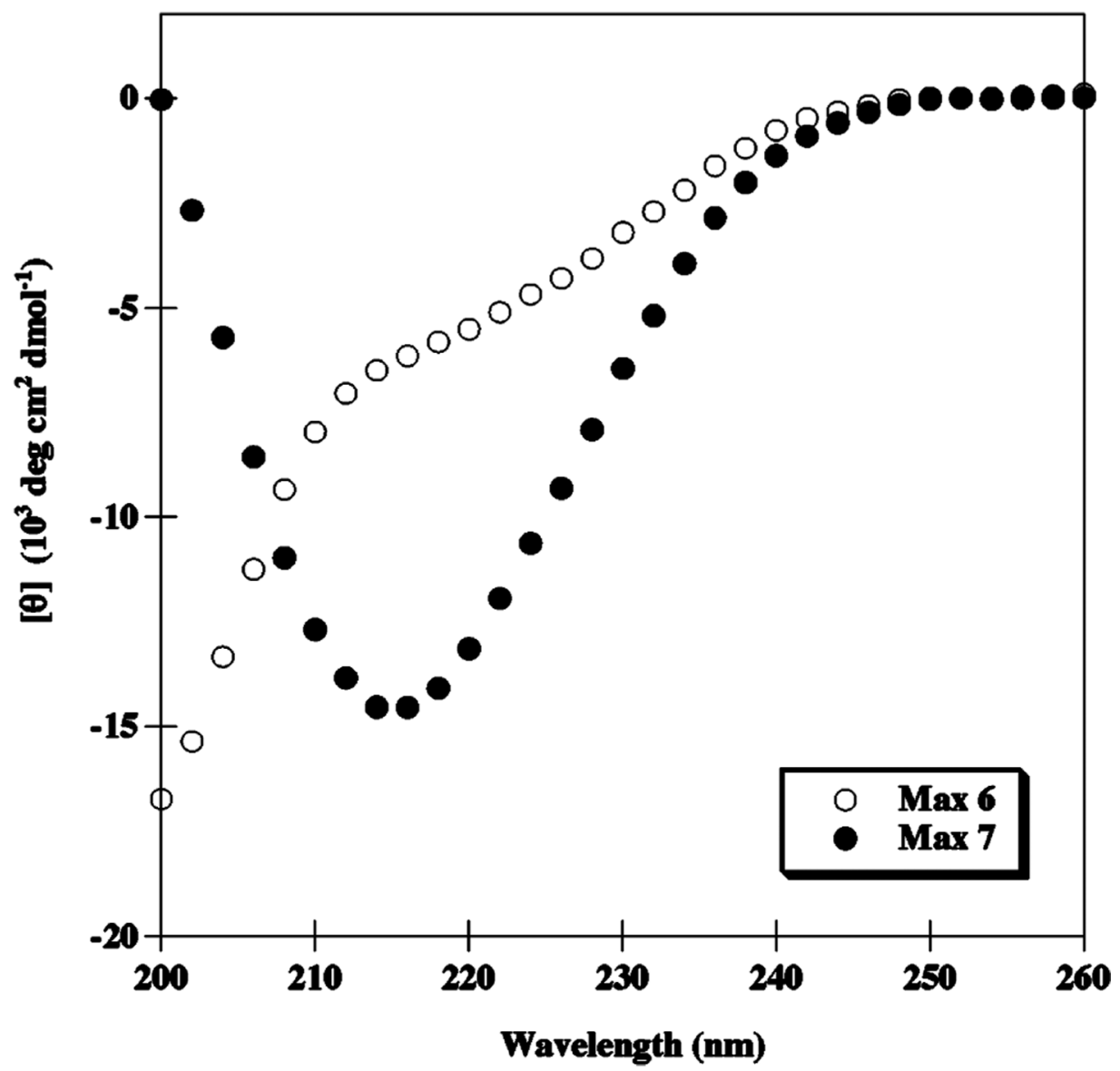

Figure 2.

CD spectra of $150 \mu \mathrm{M}$ solutions of MAX6 and MAX7 at pH 9.0 (125 mM borate and $10 \mathrm{mM}$ $\left.\mathrm{NaCl}, 37^{\circ} \mathrm{C}\right)$. 


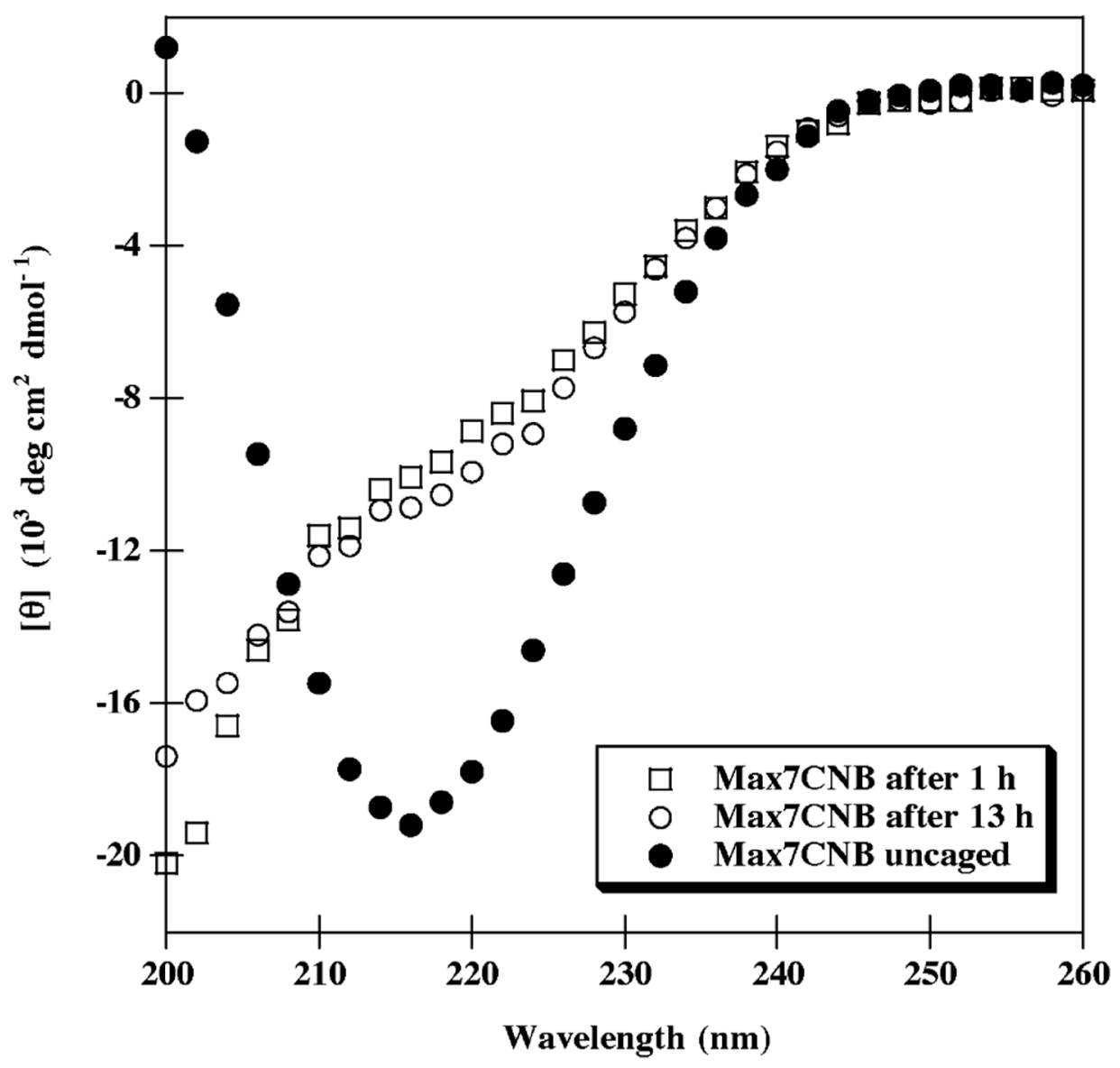

Figure 3.

CD spectra of $150 \mu \mathrm{M}$ solution of MAX7CNB (125 mM borate and $10 \mathrm{mM} \mathrm{NaCl}, \mathrm{pH} 9.0,37$ ${ }^{\circ} \mathrm{C}$ ) as a function of time. Spectra of the same solution after 1 and $13 \mathrm{~h}$ and after decaging by UV illumination are shown. 


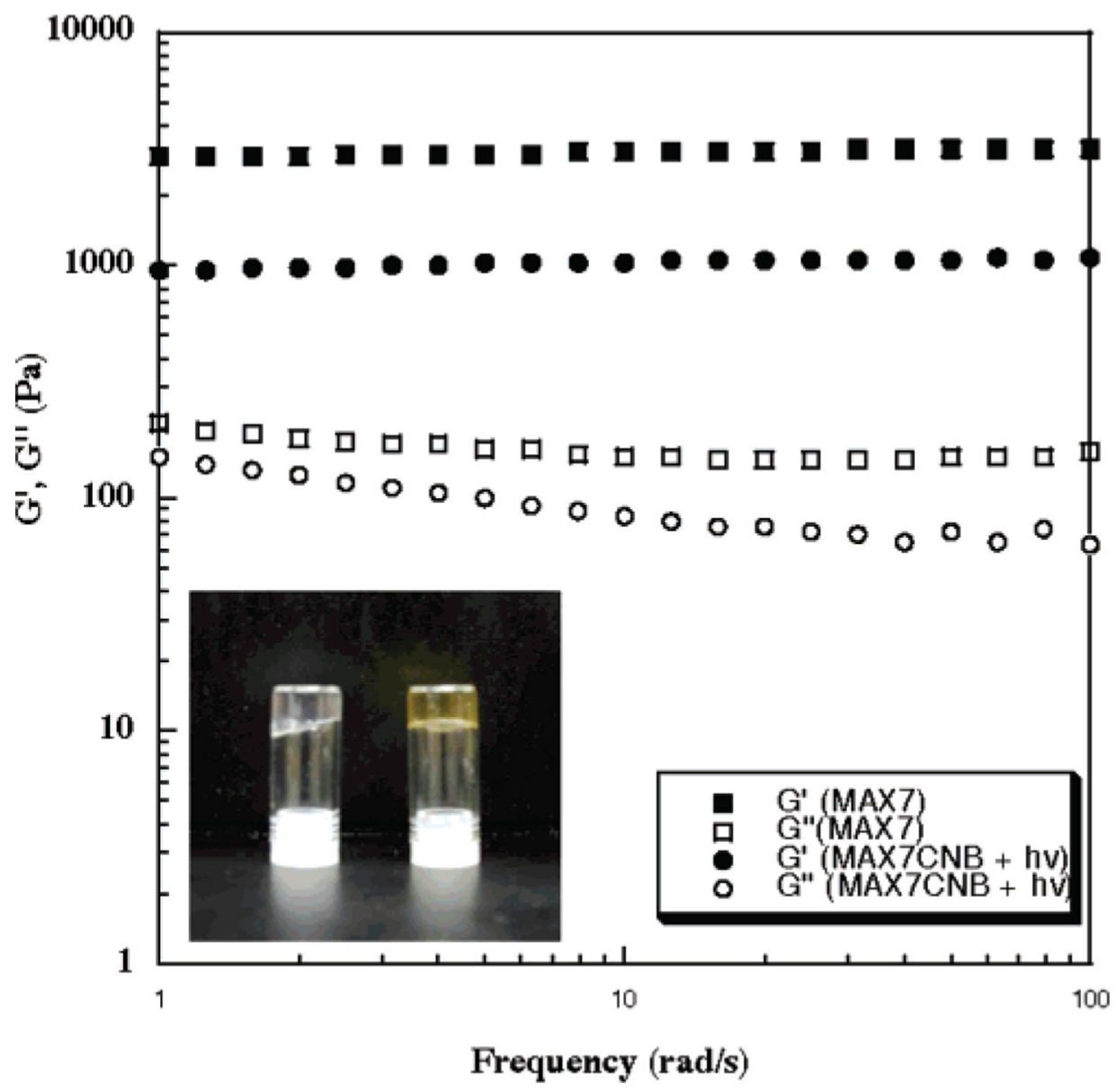

Figure 4.

Oscillatory rheology frequency sweep (1\% strain) of 2 wt \% MAX7 and MAX7CNB (after UV illumination) hydrogels. (Inset) Self-supporting MAX7 (left) and decaged MAX7CNB (right) gels. 

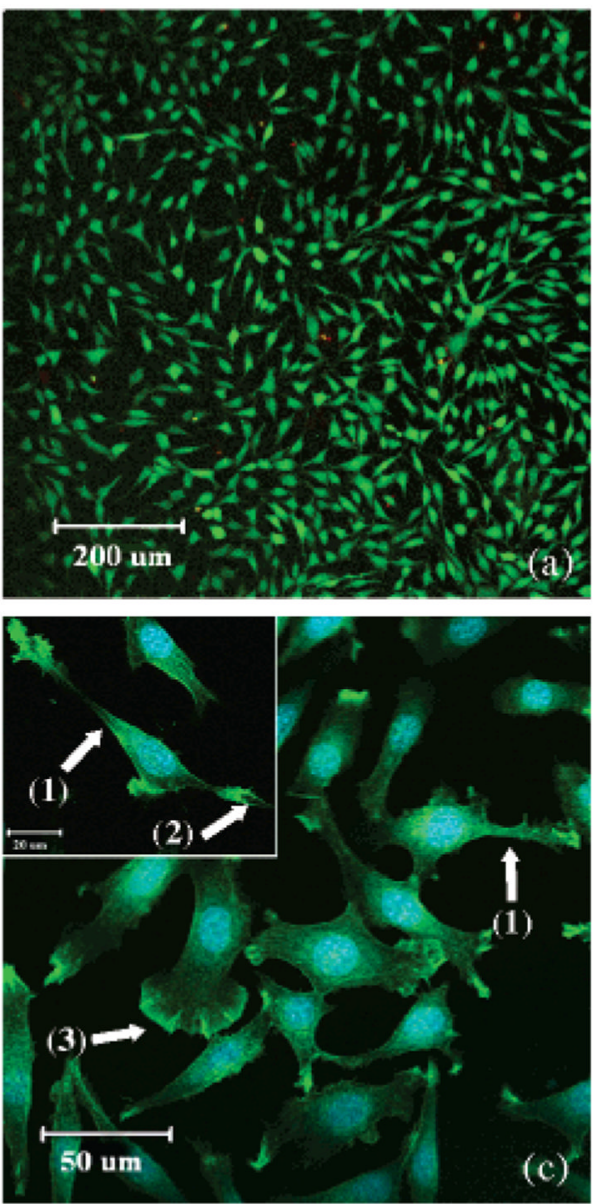
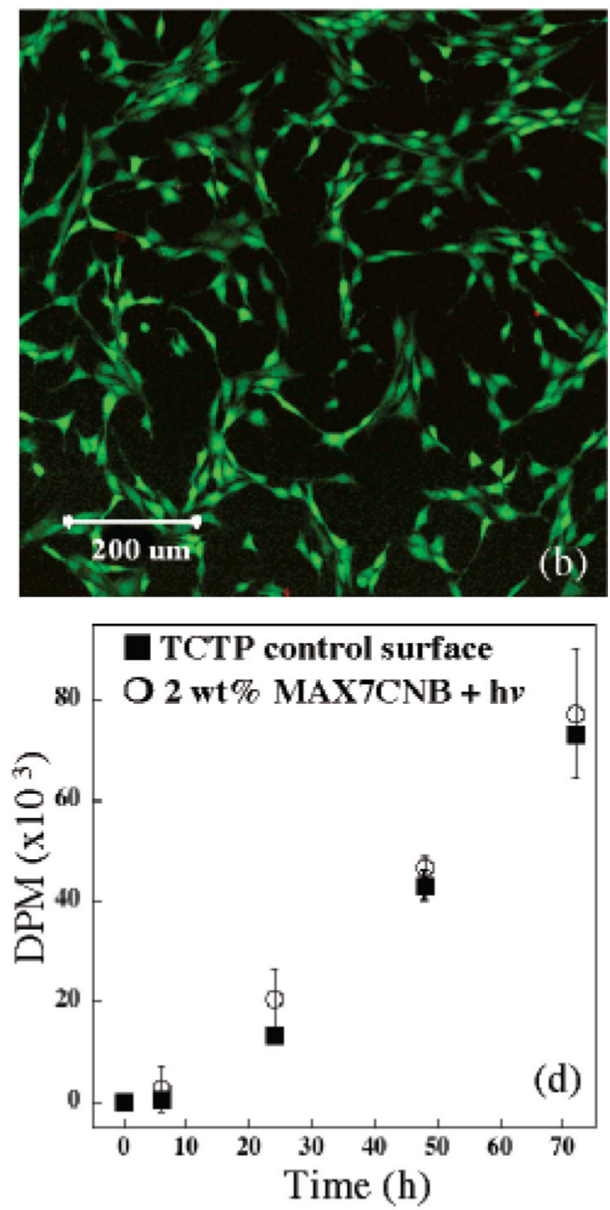

Figure 5.

(a) Live/dead assay of NIH3T3 fibroblasts seeded onto decaged MAX7CNB hydrogel after 24 h employing LSCM. Live cells fluoresce green and dead cells fluoresce red. (b) Identical assay performed on cells seeded onto a sterile borosilicate control surface. (c) LSCM image of cells at $8 \mathrm{~h}$ showing the formation of actin stress fibers (stained with a green fluorescent dye) defining the lamellipodia (1) and filopodia (2) of cells as indicated. The ruffled membrane of a migrating cell is also observed (3). Cell nuclei are stained with a blue fluorescent dye for reference. (d) Rates of cell proliferation measured by $\left[{ }^{3} \mathrm{H}\right]$ thymidine uptake of cells seeded onto 2 wt $\%$ MAX7CNB-derived gel compared to a tissue culture-treated polystyrene control surface. 